Proceedings of the Edinburgh Mathematical Society (2010) 53, 633-637 (C)

DOI:10.1017/S0013091509001394 Printed in the United Kingdom

\title{
CORRIGENDUM: AMENABILITY OF ULTRAPOWERS OF BANACH ALGEBRAS
}

\author{
MATTHEW DAWS \\ School of Mathematics, University of Leeds, \\ Leeds LS2 9JT, UK (matt.daws@cantab.net)
}

(Received 17 September 2009)

Published in Proceedings of the Edinburgh Mathematical Society 52(2) (2009), 307-338.

\begin{abstract}
Some of the results of $\S 5$ of the cited paper are incorrect: in particular, the characterization of when an algebra is ultra-amenable, in terms of a diagonal like construction, is not proved; and Theorem 5.7 is stated wrongly. The rest of the paper is unaffected. We shall show in this corrigendum that Theorem 5.7 can be corrected and that the other results of $\S 5$ are true if the algebra in question has a certain approximation property.
\end{abstract}

Keywords: Banach algebra; Arens products; ultrapower; amenability; tensor product

2010 Mathematics subject classification: Primary 46B08; 46B28

Some of the results of $\S 5$ of $[\mathbf{3}]$ are incorrect. The claim (ii) $\Rightarrow$ (i) of Proposition 5.4 implicitly assumes that $\psi_{0}$ is bounded below, but this is unproven. Hence the claim in Corollary 5.5 that, if a Banach algebra $\mathcal{A}$ is contractible, then it is ultra-amenable is also unproven. Similarly, (ii) $\Rightarrow$ (i) of Theorem 5.6 requires $\psi_{0}$ to be bounded below. The rest of the paper is unaffected. We used some of these ideas in $[4, \S 4]$, and so this is also incorrect; an erratum has been submitted.

Firstly, we deal with correcting Theorem 5.7. We say that a $C^{*}$-algebra $\mathcal{A}$ is subhomogeneous if there exists $n \in \mathbb{N}$ such that every irreducible representation of $\mathcal{A}$ has dimension at most $n$. Subhomogeneous von Neumann algebras have the special form claimed in Theorem 5.7, but this is not true for $C^{*}$-algebras (see [1, $\S$ IV.1.4] for examples). This circle of ideas was considered in $[\mathbf{7}$, Theorem 2.5] but we have been unable to follow some of the proofs (in particular, the claim that $(\mathrm{A} 4) \Rightarrow(\mathrm{R} 5)$ ) so we provide details here.

Theorem 1. Let $\mathcal{A}$ be a $C^{*}$-algebra. The following are then equivalent:

(1) $\mathcal{A}$ is ultra-amenable;

(2) $\mathcal{A}^{\prime \prime}$ is amenable; 
(3) $\ell^{\infty}(\mathcal{A}, I)$ is amenable for any index set $I$;

(4) $\mathcal{A}$ is subhomogeneous.

Proof. The original argument using the approximation property in $[\mathbf{3}]$ is correct and shows $(1) \Rightarrow(2)$. Similarly, as argued in [3] (see also [7]), if (2) holds, then $\mathcal{A}^{\prime \prime}$ has the form

$$
\mathcal{A}^{\prime \prime}=\sum_{k=1}^{n} L^{\infty}\left(X_{k}\right) \otimes \mathbb{M}_{n_{k}}
$$

where, for each $k, X_{k}$ is a measure space and $n_{k} \in \mathbb{N}$. Notice that if $\mathcal{A}^{\prime \prime}$ is of this form, then, following [7], it is elementary to see that so is $l^{\infty}\left(\mathcal{A}^{\prime \prime}, I\right)$ for any index set $I$. This does imply that $\mathcal{A}$ is subhomogeneous [1, Proposition IV.1.4.6] but not that $\mathcal{A}$ has the form originally claimed in [3, Theorem 5.7].

However, there is an algebraic characterization of when $C^{*}$-algebras are subhomogeneous (see $[\mathbf{5}, \S 3.6]$ or $[\mathbf{1}, \S I V .1 .4 .5]$ ). The algebra $\mathbb{M}_{n}$ is of dimension $n^{2}$ and so, for any $r>n^{2}$, we have

$$
\sum_{\sigma \in S_{r}} \epsilon_{\sigma} x_{\sigma(1)} \cdots x_{\sigma(r)}=0
$$

for any $x_{1}, \ldots, x_{r} \in \mathbb{M}_{n}$ (this is readily seen by taking a basis). Here $S_{r}$ is the symmetric group and $\epsilon: S_{r} \rightarrow\{ \pm 1\}$ the signature. Let $r(n)$ be the smallest $r$ for which this holds for $\mathbb{M}_{n}$. Then [5, Lemma 3.6.2] shows that $r(n) \geqslant r(n-1)+2$ (see [1, $\S$ IV.1.4.5] and references therein for better estimates). As irreducible representations separate the points of a $C^{*}$-algebra $\mathcal{A}$, we conclude that the following statements are equivalent:

(i) any irreducible representation of $\mathcal{A}$ is of dimension at most $n$;

(ii) for any $x_{1}, \ldots, x_{r(n)} \in \mathcal{A}$, identity (1) holds for $r=r(n)$.

Indeed, the only unclear issue is why (ii) cannot hold if $\pi: \mathcal{A} \rightarrow \mathcal{B}(H)$ is irreducible, with $H$ infinite dimensional. However, $\pi(\mathcal{A})$ is then strongly dense in $\mathcal{B}(H)$, and $\mathbb{M}_{n+1}$ is a subalgebra of $\mathcal{B}(H)$, which is enough to show that (ii) fails.

It is clear that condition (ii) passes to subalgebras and, with a little thought, it is seen to pass to ultrapowers as well. Thus, if $(2)$ holds, $\ell^{\infty}(\mathcal{A}, I)$ is subhomogeneous, and hence $\mathcal{A}$ is subhomogeneous, showing (4). It is reasonably easy to show that $\ell^{\infty}(\mathcal{A}, I)$ is thus nuclear [2, Proposition 2.7.7], or follow [7, Theorem 2.5] for a direct argument that $\ell^{\infty}(\mathcal{A}, I)$ is thus amenable. As amenability passes to quotients, $(3) \Rightarrow(1)$ is clear. Finally, if (4) holds, then any ultrapower of $\mathcal{A}$ is subhomogeneous and hence amenable, showing (1).

We erroneously claimed in [4] that (1) and (3) are equivalent for any Banach algebra $\mathcal{A}$. It would be interesting to know if this is true.

We shall now improve [3, Proposition 4.7] and show that the map $\psi_{0}$ is indeed bounded below for a wide class of Banach algebras $\mathcal{A}$. We leave open whether this holds for all $\mathcal{A}$ (which seems unlikely). It seems possible that similar, but stronger, conditions could characterize when $\mathcal{A}$ is ultra-amenable, but we shall not pursue this here. 
Let $E$ and $F$ be Banach spaces, and let $\mathcal{U}$ be an ultrafilter on an index set $I$. As in [3], we shall suppose that $\mathcal{U}$ is countably incomplete. Recall from $[\mathbf{3}, \S 4]$ the map $\psi_{0}:(E)_{\mathcal{U}} \hat{\otimes}(F)_{\mathcal{U}} \rightarrow(E \hat{\otimes} F)_{\mathcal{U}}$, defined on elementary tensors by

$$
\psi_{0}\left(\left(x_{i}\right) \otimes\left(y_{i}\right)\right)=\left(x_{i} \otimes y_{i}\right) \quad\left(\left(x_{i}\right) \in(E)_{\mathcal{U}},\left(y_{i}\right) \in(F) \mathcal{U}\right) .
$$

For the following, we recall that [6, Theorem 9.1] characterizes, in terms of local properties, when an ultrapower has the (bounded) approximation property.

Theorem 2. If $(E)_{\mathcal{U}}$ has the approximation property, then $\psi_{0}$ is an injection for any $F$.

Proof. Let $\tau \in(E)_{\mathcal{U}} \hat{\otimes}(F)_{\mathcal{U}}$ have representation

$$
\tau=\sum_{n=1}^{\infty} x_{n} \otimes y_{n}
$$

with

$$
\sum_{n}\left\|x_{n}\right\|\left\|y_{n}\right\|<\infty
$$

If $(E)_{\mathcal{U}}$ has the approximation property then, by [9, Proposition 4.6], if $\tau \in(E)_{\mathcal{U}} \hat{\otimes}(F)_{\mathcal{U}}$ is non-zero, there exist $\mu \in(E)_{\mathcal{U}}^{\prime}$ and $\lambda \in(F)_{\mathcal{U}}^{\prime}$ with

$$
0 \neq\langle\mu \otimes \lambda, \tau\rangle=\sum_{n=1}^{\infty}\left\langle\mu, x_{n}\right\rangle\left\langle\lambda, y_{n}\right\rangle .
$$

As we only care about the value of $\mu$ on the countable set $\left\{x_{n}\right\}$, by [6, Corollary 7.5] we may suppose that $\mu \in\left(E^{\prime}\right) \mathcal{U}$ and, similarly, that $\lambda \in\left(F^{\prime}\right) \mathcal{U}$; say $\mu=\left(\mu_{i}\right)$ and $\lambda=\left(\lambda_{i}\right)$. Pick representatives $x_{n}=\left(x_{n}^{(i)}\right)$ and $y_{n}=\left(y_{n}^{(i)}\right)$ so that, by absolute convergence,

$$
\langle\mu \otimes \lambda, \tau\rangle=\lim _{i \rightarrow \mathcal{U}} \sum_{n=1}^{\infty}\left\langle\mu_{i}, x_{n}^{(i)}\right\rangle\left\langle\lambda_{i}, y_{n}^{(i)}\right\rangle=\left\langle\left(\mu_{i} \otimes \lambda_{i}\right), \psi_{0}(\tau)\right\rangle .
$$

Hence we must have that $\psi_{0}(\tau) \neq 0$.

Consequently, [3, Corollary 5.5] correctly shows that if $\mathcal{A}$ is a contractible Banach algebra with the approximation property, then $\mathcal{A}$ is ultra-amenable. However, a result of Selivanov [8, Theorem 4.1.5] shows that under these conditions, $\mathcal{A}$ is already the finite sum of full matrix algebras!

It is worth pointing out what can go wrong here (and hence the exact mistake in the proof of $[\mathbf{3}$, Proposition 5.4]). If $\mathcal{A}$ is contractible, then we can find $\tau \in \mathcal{A} \hat{\otimes} \mathcal{A}$ with $a \cdot \tau=\tau \cdot a$ and $\Delta(\tau) a=a$ for $a \in \mathcal{A}$. We can then treat $\tau$ as a member of $(\mathcal{A})_{\mathcal{U}} \hat{\otimes}(\mathcal{A})_{\mathcal{U}}$, and we have that $a \cdot \psi_{0}(\tau)=\psi_{0}(\tau) \cdot a$ for $a \in(\mathcal{A})_{\mathcal{U}}$. As $\psi_{0}$ is an $(\mathcal{A})_{\mathcal{U}}$-module homomorphism, $\psi_{0}(a \cdot \tau-\tau \cdot a)=0$ for any $a \in(\mathcal{A})_{\mathcal{U}}$. However, if $\psi_{0}$ might fail to be injective, then this is not useful.

The following improves [3, Proposition 4.7], as a result of Grothendieck [9, Corollary 5.51] shows that a reflexive Banach space with the approximation property automatically has the metric approximation property. 
Theorem 3. If $(E)_{\mathcal{U}}$ has the bounded approximation property, then $\psi_{0}$ is bounded below.

Proof. Let $(E)_{\mathcal{U}}$ have the bounded approximation property with bound $M$, so, by (the obvious generalization of) [9, Theorem 4.14], the embedding $(E)_{\mathcal{U}} \hat{\otimes}(F)_{\mathcal{U}} \rightarrow$ $\mathcal{F}\left((E)_{\mathcal{U}},(F)_{\mathcal{U}}^{\prime}\right)^{\prime}$ is bounded below by $M^{-1}$. Here $\mathcal{F}\left((E)_{\mathcal{U}},(F)_{\mathcal{U}}^{\prime}\right)$ is the collection of finiterank operators $(E)_{\mathcal{U}} \rightarrow(F)_{\mathcal{U}}^{\prime}$, given the operator norm.

Let $\tau \in(E)_{\mathcal{U}} \hat{\otimes}(F)_{\mathcal{U}}$ have representative

$$
\tau=\sum_{n=1}^{\infty} x_{n} \otimes y_{n} .
$$

For $\epsilon>0$, we can find $T \in \mathcal{F}\left((E)_{\mathcal{U}},(F)_{\mathcal{U}}^{\prime}\right)$ with $\|T\| \leqslant M+\epsilon$ and $|\langle T, \tau\rangle| \geqslant\|\tau\|$. We work with the finite-rank operators so that we can pick a representative

$$
T=\sum_{k=1}^{N} \mu_{k} \otimes \lambda_{k}
$$

for some $\left(\mu_{k}\right) \subseteq(E)_{\mathcal{U}}^{\prime}$ and $\left(\lambda_{k}\right) \subseteq(F)_{\mathcal{U}}^{\prime}$. Thus

$$
\langle T, \tau\rangle=\sum_{n=1}^{\infty} \sum_{k=1}^{N}\left\langle\mu_{k}, x_{n}\right\rangle\left\langle\lambda_{k}, y_{n}\right\rangle .
$$

Let $G$ be the closed span of $\left\{x_{n}\right\}$, so, by [6, Corollary 7.5], as $G$ is finite dimensional, we can find a contraction $\phi: \operatorname{lin}\left\{\mu_{k}\right\} \rightarrow\left(E^{\prime}\right)_{\mathcal{U}}$ such that

$$
\left\langle\phi\left(\mu_{k}\right), x\right\rangle=\left\langle\mu_{k}, x\right\rangle \quad(1 \leqslant k \leqslant N, x \in G) .
$$

It is not hard to see that

$$
T_{0}=\sum_{k=1}^{N} \phi\left(\mu_{k}\right) \otimes \lambda_{k}
$$

then satisfies $\left\|T_{0}\right\| \leqslant M+\epsilon$ and $\left\langle T_{0}, \tau\right\rangle=\langle T, \tau\rangle$. In other words, we can assume that $\mu_{k} \in\left(E^{\prime}\right)_{\mathcal{U}}$ for each $k$; analogously, we may also assume that $\lambda_{k} \in\left(F^{\prime}\right) \mathcal{U}$ for each $k$.

So, pick representatives $\mu_{k}=\left(\mu_{k}^{(i)}\right)$ and $\lambda_{k}=\left(\lambda_{k}^{(i)}\right)$ and, for each $i$, let

$$
T_{i}=\sum_{k=1}^{N} \mu_{k}^{(i)} \otimes \lambda_{k}^{(i)} \in \mathcal{F}\left(E, F^{\prime}\right) .
$$

As $\mathcal{U}$ is countably incomplete, we can find a sequence $\left(\epsilon_{i}\right)$ of strictly positive reals such that $\lim _{i \rightarrow \mathcal{U}} \epsilon_{i}=0$. For each $i$, pick $y_{i} \in E$ with $\left\|y_{i}\right\| \geqslant 1$ and $\left\|T_{i}\left(y_{i}\right)\right\| \geqslant\left\|T_{i}\right\|-\epsilon_{i}$. Let $y=\left(y_{i}\right)$ so $\|y\|=1$ and $T(y)=\left(T_{i}\left(y_{i}\right)\right)$ so that

$$
\lim _{i \rightarrow \mathcal{U}}\left\|T_{i}\right\|=\lim _{i \rightarrow \mathcal{U}}\left\|T_{i}\right\|-\epsilon_{i} \leqslant \lim _{i \rightarrow \mathcal{U}}\left\|T_{i}\left(y_{i}\right)\right\|=\|T(y)\| \leqslant(M+\epsilon) .
$$


Finally, a calculation shows that

$$
\langle T, \tau\rangle=\left\langle\left(T_{i}\right), \psi_{0}(\tau)\right\rangle
$$

where $\left(T_{i}\right) \in\left(\mathcal{B}\left(E, F^{\prime}\right)\right)_{\mathcal{U}} \subseteq(E \hat{\otimes} F)_{\mathcal{U}}^{\prime}$. We conclude that $\left\|\psi_{0}(\tau)\right\| \geqslant\|\tau\|(M+\epsilon)^{-1}$, so that $\psi_{0}$ is bounded below by $M^{-1}$.

This shows that [3, Theorem 5.6] does give a correct characterization of ultra-amenability for Banach algebras $\mathcal{A}$ whose ultrapowers have the bounded approximation property. This includes, for example, algebras of the form $L^{1}(G)$ for a locally compact group $G$.

Acknowledgements. I thank Seytek Tabaldyev, who brought the problems in [4] (and hence also in [3]) to my attention, Volker Runde for pointing out the error in Theorem 5.7, and both Volker Runde and the authors of [7] for suggesting that I look at polynomial identities as a way to fix Theorem 5.7.

\section{References}

1. B. BlackADAR, Operator algebras. Theory of $C^{*}$-algebras and von Neumann algebras. (Springer, 2006).

2. M. Brown AND N. OzAWA, $C^{*}$-algebras and finite-dimensional approximations (American Mathematical Society, Providence, RI, 2008).

3. M. DAws, Amenability of ultrapowers of Banach algebras, Proc. Edinb. Math. Soc. 52 (2009), 307-338.

4. M. Daws And V. Runde, Can $\mathcal{B}\left(\ell^{p}\right)$ ever be amenable?, Studia Math. 188 (2005), 151174.

5. J. Dixmier, $C^{*}$-algebras (North-Holland, Amsterdam, 1977).

6. S. HeInRICH, Ultraproducts in Banach space theory, J. Reine Angew. Math. 313 (1980), 72-104.

7. A. T.-M. Lau, R. J. Loy And G. A. Willis, Amenability of Banach and $C^{*}$-algebras on locally compact groups, Studia Math. 119 (1996), 161-178.

8. V. Runde, Lectures on amenability (Springer, 2002).

9. R. RYAN, Introduction to tensor products of Banach spaces (Springer, 2002). 